\title{
Vision-threatening diabetic retinopathy necessitating vitrectomy in a tertiary care hospital in coastal Karnataka, South India
}

\author{
Narasimha Vasista Reddy, Yogish Subraya Kamath, Lavanya G. Rao, \\ Krishna Addoor Rao, Shailaja Bhat Shenoy, Sulatha V. Bhandary \\ Department of Ophthalmology, Kasturba Medical College-Manipal, Manipal \\ Academy of Higher Education, Karnataka, India
}

\begin{abstract}
Background: The proportion of diabetic patients having vision-threatening diabetic retinopathy and those needing to undergo vitrectomy was documented.

Methods: A cross-sectional observational study was conducted at a tertiary care hospital in coastal Karnataka, South India. All patients with diabetes mellitus visiting the ophthalmology department in the study period were screened for retinopathy, and the data regarding the presence of maculopathy and proliferative retinopathy were included as vision-threatening retinopathy.

Results: Of the 1,435 diabetic patients included, 38.4\% had retinopathy changes due to diabetes, with $8.71 \%$ having vision-threatening retinopathy and $1.81 \%$ needing vitrectomy.

Conclusion: Although the proportion of vision-threatening retinopathy is small, the rapidly increasing diabetic population requires the setting up of more resources for tackling this condition, at least at the tertiary levels of the health-care system. However, considering the increased human and economic resources involved in setting up vitrectomy units, more emphasis on strengthening screening programs for early detection and referral to reduce the progression of retinopathy to advanced stages would be appropriate.
\end{abstract}

Keywords: diabetic retinopathy, India, proliferative, tertiary care centers, vitrectomy, vitreoretinopathy

\section{Introduction}

Diabetic retinopathy (DR) accounts for $1 \%$ of the blindness in the world. ${ }^{1}$ Visual loss results from proliferative diabetic retinopathy (PDR) with vitreous hemorrhage, PDR with tractional retinal detachment (TRD), or macular edema seen in any stage. ${ }^{2}$ Such stages have been termed sight or vision-threatening diabetic

Correspondence: Dr. Yogish Subraya Kamath, Associate Professor, Department of Ophthalmology, Kasturba Medical College- Manipal, Manipal Academy of Higher Education, Madhav Nagar, Manipal-576104, Karnataka, India.

E-mail: dryogishkamath@yahoo.co.in 
retinopathy (VTDR). VTDR can be managed by methods including laser photocoagulation, anti-vascular endothelial growth factor (anti-VEGF) injections, and surgical techniques involving vitrectomy., ${ }^{2,3}$ Although the former two modalities can be done as an outpatient procedure or in any sterile ophthalmic operating theater, vitreoretinal surgery can only be performed in a specialized operating room with specific and expensive equipment. This study attempts to document the number of VTDR patients who required vitrectomy at a tertiary care hospital in coastal Karnataka, South India. An understanding of the number of VTDR patients requiring vitrectomy would enable proper infrastructural planning and budget allocation, in an effort to tackle the burden of DR.

\section{Methods}

This was a cross-sectional hospital-based study conducted in a tertiary care multispecialty hospital in coastal Karnataka, South India, between November 2011 and April 2013.

The study was conducted in accordance with the Declaration of Helsinki. Institutional ethical committee was taken prior to the commencement of the study.

All patients with diabetes mellitus visiting the ophthalmology department were included. Diabetes was defined as fasting blood glucose of $>110 \mathrm{mg} \%$ and/ or postprandial glucose of $>140 \mathrm{mg} \%$ and/or glycated hemoglobin (HbA1C) of $>6.5 \%$ and/or a history of treatment with antidiabetic medication. An informed consent of the patients was obtained before including them in the study.

All the patients underwent an ophthalmological evaluation that included an assessment of visual acuity with the Snellen chart, slit lamp biomicroscopic evaluation of the anterior segment, and intraocular pressure evaluation. Patients then underwent pupillary dilation with tropicamide $1 \%$ eye drops. The retinal evaluation was done by an ophthalmologist using $+90 \mathrm{D}$ lens on a slit lamp biomicroscope and indirect ophthalmoscopy in some cases. All patients with evidence of any retinopathy were examined by ophthalmologists specializing in retinal diseases. The DR classification was adopted as per the modified Airlie House classification system used in Early Treatment Diabetic Retinopathy Study. Macular edema that was clinically significant was included as maculopathy. The details of investigations and any subsequent ophthalmological interventions were retrieved and analyzed from the medical records of patients.

VTDR was defined as the presence of any nonproliferative diabetic retinopathy (NPDR) with macular edema and all cases of PDR. The patients in whom vitrectomy was indicated included those with

- Nonclearing vitreous hemorrhage of one-month duration, if they had not undergone pan-retinal photocoagulation (PRP) previously; 
- Non-clearing vitreous hemorrhage of three-month duration, if they had undergone PRP previously;

- Recurrent vitreous hemorrhage;

- Progressive fibrovascular proliferation;

- TRD involving macula;

- Macular edema associated with taut posterior hyaloid membrane; and

- Neovascular glaucoma as a consequence of PDR with some visual potential.

Patients with vitreous hemorrhage, TRD, macular edema, and neovascular glaucoma due to causes other than diabetes were excluded.

The data collected were analyzed using SPSS, version 16.0. For statistical analysis, data were summarized using frequency and percentage for categorical variables.

Table 1. The number and percentage of patients with various types of retinopathy

\begin{tabular}{|l|l|l|}
\hline Type of retinopathy & Number of patients $(\mathbf{n})$ & Percentage \\
\hline Mild NPDR & 116 & 8.08 \\
\hline Moderate NPDR & 191 & 13.31 \\
\hline Severe NPDR & 96 & 6.68 \\
\hline Very severe NPDR & 23 & 1.60 \\
\hline NPDR with maculopathy & 49 & 3.41 \\
\hline PDR & 63 & 4.39 \\
\hline PDR + maculopathy & 13 & 0.9 \\
\hline Total DR & 551 & 38.4 \\
\hline No DR & 884 & 61.6 \\
\hline Total patients & 1,435 & 100 \\
\hline & & \\
\hline
\end{tabular}

\section{Results}

There were 1,435 diabetic patients included in the study, of whom $66.57 \%$ were males. Majority of them (88.49\%) were in the age group of 31 to 70 years. DR changes were absent in $61.60 \%$ patients. The number and percentile of patients with the various types of DR are given in Table 1. 
Vision-threatening diabetic retinopathy in coastal Karnataka

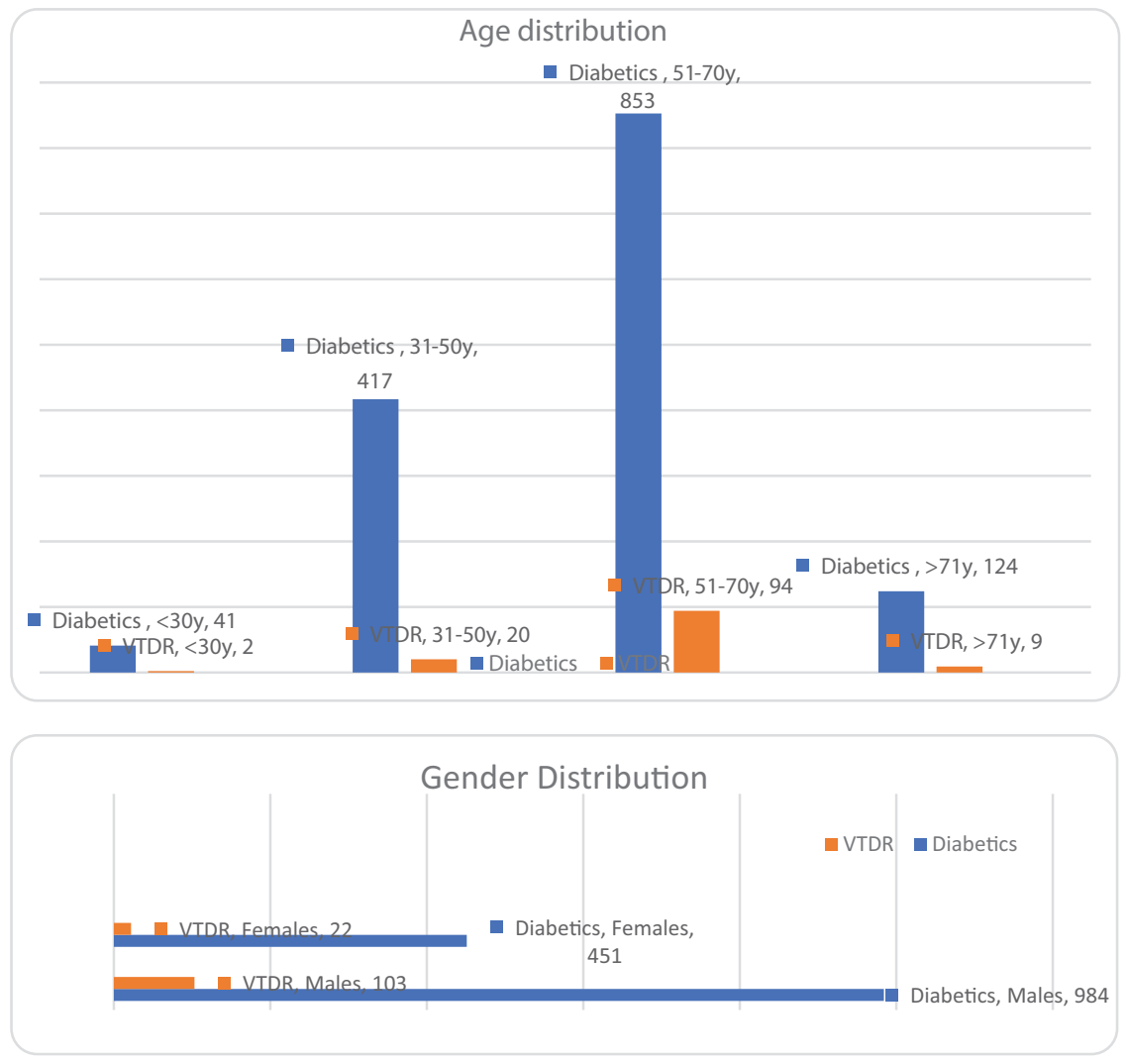

Fig. 1. Age and gender distribution of patients with VTDR.

The total number of patients with VTDR were 125 (8.71\%). Figure 1 depicts the comparison of age and gender distribution of the VTDR patients among diabetics. Among the patients with VTDR, vitrectomy was indicated and advised in 26 patients (1.81\%).

\section{Discussion}

This study documents the number of VTDR patients requiring surgical intervention. With the increasing numbers of patients with diabetes mellitus in the world, data on the regional prevalence of DR become important for planning the management strategy. Although the initial stages of DR require merely observation and systemic glycemic control, advanced stages of vision-threatening retinopathy require more intensive management. ${ }^{2}$ The major modalities of 
managing PDR include retinal photocoagulation, intravitreal anti-VEGF injections, and, in certain cases, vitreoretinal surgery. ${ }^{4}$ The infrastructural and human resources needed for managing advanced retinopathy are limited. The expenses involved in the establishment of the needed resources for screening and management of DR are also high. Hence, a reliable estimation of the disease burden would help in the proper allocation of the limited resources to ensure optimal utilization. Only a small portion of patients would need surgical intervention for the management of VTDR, especially in cases of vitreous hemorrhage or retinal detachment. The hospital-based setup of our study was a limiting factor in determining the prevalence of VTDR. However, we felt that data regarding patients with VTDR requiring surgical therapy were more likely to be obtained from a tertiary care hospital providing vitreoretinal services.

In our study, DR was seen in $38.4 \%$ of diabetic patients. This was marginally higher than a $33.9 \%$ prevalence in another hospital-based study from Western India ${ }^{5}$ and considerably higher than $20.4 \%$ reported in a population-based prevalence study in South India. ${ }^{6}$ However, it was closer to the $45 \%$ prevalence reported in the South Asian community settled in the United Kingdom by Raymond et al. ${ }^{7}$

PDR was seen in $5.29 \%$ of diabetics in this study with NPDR in $33.08 \%$ patients. The proportion of retinopathy detection was higher than other studies and there were more NPDR cases detected in our study. 5,6 The difficulty in the detailed clinical screening of retinopathy in population-based studies may affect the accurate staging, especially of early stages. This factor may affect the prevalence values. ${ }^{8}$ The use of fundus photography and evaluation can overcome this hurdle but is expensive for use in developing countries. ${ }^{6}$ Our study probably was able to detect more retinopathy due to a detailed stereoscopic fundus evaluation of all patients, as well as the availability of confirmation by a specialist in doubtful cases. Poor retinopathy detection and management have been attributed to the lack of a specialist retina service. ${ }^{9}$

Many of the patients visiting our hospital had other systemic diabetic complications. The presence of retinopathy is known to be higher in the presence of coexisting microvasculopathies of other systems. ${ }^{10}$ Hence, referral from these departments may also have accounted for a higher prevalence in our study.

We found that VTDR was present in $8.71 \%$ of the diabetics. Most of the patients with VTDR (91.2\%) were in the age group of 31 to 70 years and $81.4 \%$ were males. Nearly $60 \%$ of these patients were in the working age group of 25 to 60 years. This implies that not only is there a threat of severe visual loss in almost $5.15 \%$ of all the diabetic patients in our study but that it will affect the potential breadwinners of their families. DR is known to be the leading cause of preventable blindness in the working adult population in the United States as well. ${ }^{11}$ 
Although the total DR percentage in our study is similar to the study by Raymond et al., the proportion of VTDR is lesser $(8.71 \%$ compared with $16 \%$ noted by Raymond et al. $)^{7}$ This may be attributed to the exclusion of severe NPDR without significant macular edema as vision threatening in our study.

We observed more males presenting with DR (68.57\%) as well as VTDR (81.4\%) compared with females. Other epidemiological studies ${ }^{12,13}$ and reviews ${ }^{14}$ have also observed a male predominance in DR.

Among the 125 patients with VTDR, vitrectomy was indicated in 26 patients. These accounted for nearly $1.81 \%$ of our diabetic study population. The benefits of vitrectomy in PDR were initially suggested by the Diabetic Retinopathy Vitrectomy Study (DRVS), and subsequent studies have strengthened this concept. ${ }^{15,16}$ The technological advancements in surgical approach are probably the main factors responsible for this improved outcome. ${ }^{16}$ However, such improved operating systems demand more elaborate and stringent operation theatres and surgical instruments.

In recent years, though, there has also been a decline in the rates of vitrectomy needed for DR management in some countries, due to an overall decrease in advanced stages of diabetic eye disease. This has been achieved due to an extensive and meticulous screening, early detection, and treatment program by these countries. ${ }^{17}$ In addition to this, better dietary habits, lifestyle modification, and systemic therapy for glycemic control have probably contributed to lessening the rates of end-organ damage due to diabetes, including retinopathy. ${ }^{18}$

Hence, in countries with fewer resources but a high load of diabetic patients, systemic control of the disease by dietary and medical means should be emphasized. The improvement of screening programs for early detection of retinopathy patients would avoid progression to advanced stages. The use of teleophthalmology and telemedicine services can improve this endeavor. ${ }^{19}$ Referral services to tertiary care centers providing integrated and complete care for diabetes and its complications can then be facilitated.

\section{References}

1. Pascolini D, Mariotti SP. Global estimates of visual impairment: 2010. Br J Ophthalmol. 2012;96:614-618.

2. Fong DS, Aiello L, Gardner TW, et al. Retinopathy in diabetes. Diabetes Care. 2004;27:S84-S87.

3. Heng LZ, Comyn O, Peto T, et al. Diabetic retinopathy: pathogenesis, clinical grading, management and future developments. Diabet Med. 2013;30:640-650.

4. Mohamed QA, Ross A, Chu CJ. Diabetic retinopathy (treatment). BMJ Clin Evid. 2011;pii:0702. PMID: 21609511 PMCID: PMC3217806.

5. Ramavat PR, Ramavat MR, Ghugare BW, Vaishnav RG, Joshi MU. Prevalence of diabetic retinopathy in Western Indian Type 2 Diabetic population: a hospital-based cross-sectional study. J Clin Diagn Res. 2013;7:1387-1390. 
6. Namperumalsamy P, Nirmalan PK, Ramasamy K. Developing a screening program to detect sight-threatening diabetic retinopathy in South India. Diabetes Care. 2003;26:1831-1835.

7. Raymond NT, Varadhan L, Reynold DR, et al. Higher prevalence of retinopathy in diabetic patients of South Asian ethnicity compared with white Europeans in the community: a crosssectional study. Diabetes Care. 2009;32:410-415.

8. Ruta LM, Magliano DJ, Lemesurier R, Taylor HR, Zimmet PZ, Shaw JE. Prevalence of diabetic retinopathy in Type 2 diabetes in developing and developed countries. Diabet Med. 2013;30:387-398.

9. Gilbert CE, Babu RG, Gudlavalleti AS, et al. Eye care infrastructure and human resources for managing diabetic retinopathy in India: The India 11-city 9-state study. Indian J Endocrinol Metab. 2016;20:S3-S10.

10. Tomita M, Kabeya $Y$, Okisugi $M$, et al. Diabetic microangiopathy is an independent predictor of incident diabetic foot ulcer. J Diabetes Res. 2016;2016:5938540. doi: 10.1155/2016/5938540.

11. Mohamed Q, Gillies MC, Wong TY. Management of diabetic retinopathy: a systematic review. JAMA. 2007;298:902-916.

12. Rema M, Premkumar S, Anitha B, Deepa R, Pradeepa R, Mohan V. Prevalence of diabetic retinopathy in urban India: the Chennai Urban Rural Epidemiology Study (CURES) eye study, I. Invest Ophthalmol Vis Sci. 2005;46:2328-2333.

13. Raman R, Rani PK, Reddi Rachepalle S, et al. Prevalence of diabetic retinopathy in India: Sankara Nethralaya Diabetic Retinopathy Epidemiology and Molecular Genetics Study report 2. Ophthalmology. 2009;116:311-318.

14. Ozawa GY, Bearse MA Jr, Adams AJ. Male-female differences in diabetic retinopathy? Curr Eye Res. 2015;40:234-246.

15. Early vitrectomy for severe proliferative diabetic retinopathy in eyes with useful vision. Results of a randomized trial--Diabetic Retinopathy Vitrectomy Study Report 3. The Diabetic Retinopathy Vitrectomy Study Research Group. Ophthalmology. 1988 Oct;95(10):1307-1320.

16. Gupta B, Sivaprasad S, Wong R, et al. Visual and anatomical outcomes following vitrectomy for complications of diabetic retinopathy: the DRIVE UK study. Eye (Lond). 2012;26:510-516.

17. Bäcklund LB, Algvere PV, Rosenqvist U. New blindness in diabetes reduced by more than one-third in Stockholm County. Diabet Med. 1997;14:732-740.

18. Diabetes Control and Complications Trial Research Group, Nathan DM, Genuth S, Lachin J, et al. The effect of intensive treatment of diabetes on the development and progression of long-term complications in insulin-dependent diabetes mellitus. N Engl J Med. 1993;329:977-986.

19. Mohan V, Deepa M, Pradeepa R, et al. Prevention of diabetes in rural India with a telemedicine intervention. J Diabetes Sci Technol. 2012;6:1355-1364. 